Voix et Images

voixetimages

\title{
Entrevue avec Yolande Villemaire
}

\section{Lucie Robert}

Volume 11, numéro 3 (33), printemps 1986

Yolande Villemaire

URI : https://id.erudit.org/iderudit/200576ar

DOI : https://doi.org/10.7202/200576ar

Aller au sommaire du numéro

\section{Éditeur(s)}

Université du Québec à Montréal

\section{ISSN}

0318-9201 (imprimé)

1705-933X (numérique)

Découvrir la revue

\section{Citer ce document}

Robert, L. (1986). Entrevue avec Yolande Villemaire. Voix et Images, 11(3), 390-405. https://doi.org/10.7202/200576ar d'utilisation que vous pouvez consulter en ligne.

https://apropos.erudit.org/fr/usagers/politique-dutilisation/ 


\title{
Entrevue avec Yolande Villemaire
}

\author{
par Lucie Robert, Université Laval
}

V. \& I. - Tu es venue à Montréal donner des ateliers d'écriture. En donnes-tu souvent?

Y. Villemaire - Il y a trois ans, j'ai créé un groupe de travail, une spirale qui s'appelle Rrose Sélavy, dont les membres sont des amies. L'année dernière, j'ai commencé à organiser des ateliers où j'invite des gens que je ne connais pas à travailler avec moi. Je le fais d'abord dans un but financier, car, en ce moment, j'habite New York et je ne peux pas y travailler. Cet atelier était le troisième. C'est vraiment merveilleux d'être payée pour faire la chose qu'on aime le plus au monde.

\section{V. \& I. - En quoi consistent ces ateliers?}

Y. Villemaire - Depuis 1979, j'ai suivi beaucoup d'ateliers, pas nécessairement de création, des ateliers reliés aux Enfants du Verseau de Marilyn Ferguson, aux «spiral information networks» comme il en existe aux ÉtatsUnis depuis les années soixante. J'ai fait à peu près tous ceux qui existent: "EST", "Relationships", "Six Days», "LRT», "Communication for Action», "Communication Workshop». Ces ateliers sont suivis très souvent par des gens qui sont dans le domaine des affaires et par ceux qui sont dans ce qu'on appelle la croissance personnelle. J'ai fait des choses ésotériques et des choses pragmatiques aussi. C'est à la suite de cela que j'ai pensé à mettre sur pied un atelier pour les gens qui écrivent, en utilisant aussi des méthodes synergétiques. Ce qui est intéressant en effet, c'est de voir comment tous ces groupes fonctionnent à partir d'une énergie positive de base. Comme j'enseigne la création depuis plusieurs années, j'ai pu recréer l'expérience et mettre au point une méthode originale. Dans les ateliers d'écriture, je mets en place une sorte de combinatoire d'énergie. D'une certaine façon, la seule chose peut-être que je sais faire comme écrivaine, c'est de me brancher. Alors je combine l'énergie des participants à la mienne. J'essaie de me brancher sur une espèce de courant qui est celui de l'inspiration, et je les branche un à un à ce courant-là, de sorte que nous allons tous dans la même direction. Mon principe de départ est que tout le monde est écrivain.

V. \& I. - Qu'est-ce qu'on vient chercher dans ces ateliers? 
Y. Villemaire - L'inspiration et le droit d'écrire. Évidemment, les gens qui s'inscrivent à un atelier d'écriture ont tous envie d'écrire, mais ils ont un doute. Ils se demandent s'ils ont le droit de le faire, s'ils peuvent prendre la parole. Ils formulent cela autrement, se demandent s'ils ont du talent, s'ils vont réussir. Pour moi, il est évident que qui que ce soit qui veut écrire, peut le faire. J'en suis tellement convaincue, que je réussis à les convaincre facilement. Je reconnais leur désir d'écrire, je leur reconnais le droit de le faire. C'est ça qu'ils viennent chercher. Dans cet atelier-ci, j'ai mis l'accent sur la matérialisation de ce désir d'écrire. C'est bien beau de produire un texte en atelier, mais il faut aussi le matérialiser à l'extérieur. Tu vois, c'est surtout des femmes qui s'inscrivent à mes ateliers. Dans les trois, ça a été la même chose: il n'y avait qu'un seul homme. Je leur racontais que moi, je n'ai jamais eu de difficulté avec l'idée d'écrire. J'ai écrit mes premiers romans à l'âge de neuf ans. Ils se passaient à Paris, ils avaient douze pages. Je ne savais pas comment écrire, mais j'avais le désir de le faire. J'écrivais en me disant que, quand je serais morte, vers l'âge de trente ans, un éditeur trouverait mes textes dans le tiroir du bas. C'est Claude Beausoleil qui m'a poussée à matérialiser. Il m'a dit que ça ne se passait pas comme cela, qu'il faut envoyer ses textes à un éditeur, à une revue... C'est ce que j'ai fait. J'ai eu des refus qui ont été une étape importante. C'est comme ça que peu à peu j'ai appris à matérialiser. Nous avions pourtant le même âge, nous allions au même collège, mais lui, il savait comment matérialiser. Moi je n'aurais jamais eu l'idée de le faire.

J'ai une alliance fondamentale avec Claude Beausoleil au niveau de l'écriture. Nous nous connaissons depuis l'âge de dix-neuf ans, nous avons vécu ensemble pendant une dizaine d'années et nous sommes restés de très grands amis. C'est une alliance qui a été très importante pour moi, qui l'est encore beaucoup. Il a fait pour moi une espèce de pont avec la matérialisation. J'ai eu besoin de ce support. Parce qu'on ne devient pas un auteur si on ne publie pas, si on n'envoie pas son texte à un éditeur. Il y a beaucoup de gens qui ignorent cela.

Les femmes qui suivent mes ateliers d'écriture ont cette attitude que j'avais. Elles ont une démarche d'écriture, elles écrivent depuis plusieurs années déjà, mais elles ne sortent pas leurs textes. Alors je leur donne une petite poussée. Dans le dernier atelier, je leur ai suggéré d'envoyer un texte à tel ou tel endroit, d'écrire pour la radio, par exemple. J'ai donné des suggestions très précises. Je sais l'importance de la publication. Une fois qu'un texte a été publié, qu'il a été diffusé, que des gens l'ont lu, qu'il circule, je peux avancer, aller ailleurs. Si j’accumule des manuscrits que je ne transmets jamais, je ne peux pas évoluer dans mon écriture.

\section{V. \& I. - Quelle est la différence avec les cours que tu donnais au cégep?}

Y. Villemaire - Je donnais le cours de français IV, qui est le cours de création. C'est un cours obligatoire. Les étudiants étaient en techniques administratives, en droit, en thanatologie... Les étudiants en lettres étaient toujours minoritaires dans les cégeps où j'ai enseigné. Remarque que c'est une difficulté mineure parce que tout le monde a envie d'écrire. C'est quelque 
chose d'absolument remarquable. Le principal problème que je rencontrais était de l'ordre des difficultés de langue, mais je travaillais beaucoup à l'oral. J'allais chercher mes étudiants par la séduction. J'avais beaucoup de plaisir à les entraîner dans des espaces de fiction. À André-Laurendeau, l'année dernière, j'avais deux groupes en Atlantide, en 30000 av. J.-C. et deux groupes au Japon en 1940. Je pouvais arriver dans une classe et me tromper d'espace de fiction. Les étudiants me ramenaient vite à la réalité. C'était amusant, mais ça demandait beaucoup d'énergie. Avec trente ou trente-cinq étudiants, le processus devient très lourd. Ils écrivaient le texte une semaine et la lecture de ces textes pouvait prendre trois heures la semaine suivante. Il ne restait pas beaucoup de temps pour les commentaires. Alors nous faisions bouger la fiction. Nous avancions dans une histoire en quatre, cinq ou six textes seulement. Les étudiants établissaient leur nom et les rapports entre les personnages. À l'époque où j'enseignais quinze heures par semaine, je devenais saturée de création. Après l'atelier que je viens de donner par exemple, j'en ai au moins pour une bonne semaine à ne pas avoir envie d'écrire. En même temps, l'enseignement me passionne. Je préfère enseigner la fiction, la création, plutôt que l'analyser.

\section{V. \& I. - Tu l'as pourtant fait pendant des années!}

Y. Villemaire - Oui. De l'âge de vingt ans à l'âge de vingt-sept ans, j'ai été vraiment passionnée par l'analyse littéraire. Certaines de ces analyses ont été très importantes. J'ai fait une thèse sur $\bar{A}$ toi pour toujours, ta Marie-Lou et j'ai eu beaucoup de plaisir à décoder l'œuvre de Michel Tremblay. J'ai travaillé Hubert Aquin. Dans la revue Cul-Q, j’ai analysé longuement French Kiss de Nicole Brossard. J'utilisais beaucoup les méthodes de Tzvetan Todorov, qui me passionnaient. J'ai même suivi des cours avec lui à Urbino, en Italie, en 1978. J'ai écrit aussi des chroniques de théâtre. Ce n'était pas vraiment de la critique, plutôt des descriptions de spectacles. A travers le théâtre, je me suis intéressée au mouvement énergétique, à ce qui se joue et qui n'est pas dans le texte, mais dans la présence des acteurs et des spectateurs. Ce qui m'a amenée ensuite aux ateliers que j'ai commencé à faire en 1979.

V. \& I. - Mis à part Belles de nuit, qui est un texte radiophonique d'ailleurs, tu n'as jamais écrit pour le théâtre. L'as-tu abandonné?

Y. Villemaire - Je n'ai jamais voulu écrire pour le théâtre. C'est une résistance que j'ai. J'ai étudié l'interprétation à l'Atelier de Sainte-Marie, bien que je n'aie jamais voulu être comédienne. J'ai toujours beaucoup aimé la peinture et le dessin. Les costumes me fascinaient. L'interprétation me paraissait hiératique. J'avais très peur de la salle. J'avais beaucoup de trac. À la fin de mes études en théâtre, je m'étais dit que je ne remonterais plus sur une scène. En 1977 , j'ai pourtant commencé à faire des performances, un peu sans y penser. Quand nous avons présenté Célébrations, au TNM, en 1979, je me suis rappelé cet engagement que j'avais pris. La panique m'est revenue. Pourtant, dans Célébrations, j'étais très à l'aise: c'était mon propre texte, un extrait de la Vie en prose. Par la suite, j'ai fait de plus en plus de performances. La 
performance n'existait pas à l'époque où j'étudiais. J'ai découvert plus tard qu'elle convenait à ce que j'avais envie de faire. J'ai pu à la longue créer ma propre forme. J'aime être présente sur une scène. J'aime être en contact avec un public. Ma peur venait d'avoir à incarner un personnage.

J'ai une relation difficile avec le théâtre. C'est une relation passionnée. Je trouve absurde qu'un auteur écrive un texte, que des comédiens le jouent. Pour moi, le théâtre est quelque chose qui inclut l'ensemble des spectateurs et des acteurs, la scène et la salle. Je suis intéressée par exemple au travail du groupe de la Veillée. À l'époque où j'écrivais ma chronique de théâtre, j'étais fascinée par la Bebitte à Roches (rushes). C'est une troupe qui n'a pas duré très longtemps, mais qui allait très loin dans la recherche de ce qu'est être présent devant des spectateurs. J'ai même fait une entrevue avec eux pour Hobo-Québec. J'allais voir plusieurs représentations du même spectacle. Ils étaient constamment en train de le transformer: ils avaient chaque fois de nouveaux costumes, de nouveaux accessoires. J'aime les spectacles fondés sur la présence. J'ai vu récemment au Brooklyn Academy of Music, Golden Windows de Robert Wilson qui écrit des textes plus près de la performance que de la structure dramatique. Il fait son propre éclairage, travaille avec ses comédiens; ses spectacles sont presque de l'ordre de la peinture.

Je n'aime pas l'idée de l'identification, de l'identité de la représentation. Le théâtre est toujours événementiel. Il doit se transformer. Le jour où je travaillerai en théâtre, je ferai quelque chose de cet ordre-là. Écrire une pièce de théâtre ne m'intéresse pas.

\section{V. \& I. - Quand tu écris tes performances, tu les fixes aussi, non?}

Y. Villemaire - Ce n'est pas la même chose. Je suis toujours l'interprète de la performance. Il n'y a pas d'actants en relation. Quand on écrit une pièce de théâtre, comme $\dot{\mathbf{A}}$ toi pour toujours, ta Marie-Lou, on doit penser aux personnages, au décor, aux costumes, au jeu, à la représentation. Dans une performance, il y a aussi une dimension visuelle, mais comme je suis l'animatrice, je peux la transformer. Il y a d'ailleurs très souvent des aspects que je ne fixe qu'au moment de la représentation. Je ne répète pas mes performances. Je construis une structure et ensuite je la présente, une seule fois. Ce n'est pas une méthode très commerciale, mais c'est quelque chose qui m'amuse. Comme la plupart des performants, je ne m'insère dans aucune forme pré-existante. Mais je ne me concentre pas vraiment sur la préparation d'une performance. La première fois que je l'ai fait, c'est au cours de l'été dernier, en préparant Rrose Sélavy, New York 1921, la performance que j’ai présentée en août dernier au Studio du Québec à New York.

V. \& I. - Qu'est-ce que c'était comme performance?

Y. Villemaire - Je suis allée chercher la source de Rrose Sélavy comme concept. Marcel Duchamp n'a pas produit beaucoup de matière, mais c'est un artiste important au niveau de la réflexion sur l'art. Il a produit ce que Louis-Philippe Hébert appellerait des machines molles, des concepts sur 
l'art. Rrose Sélavy, en réalité, c'est deux photos par Man Ray, quelques aphorismes, de l'eau de toilette et quelques petits éléments. Comme production matérielle, ce n'est pas très important, mais cela a eu un grand impact sur mon imaginaire, à cause du rose, évidemment, du "Éros c'est la vie» qu'on entend, du double «r»). À l'époque où j'écrivais la Vie en prose, j'étais à la recherche de diachronies contenant du rose. Je connaissais Marcel Duchamp pour la Mariée mise à nue par ses célibataires, même, mais je ne connaissais pas le travail sur Rrose Sélavy. C'est un ami à qui j’avais fait part de mes recherches qui m'en a parlé. Dans la Vie en prose, le personnage apparaît au début $d$ 'un chapitre et disparaît immédiatement. Puis il y a eu le groupe d'écriture. C'est devenu ensuite cette recherche sur les origines du concept. Je me suis servie de Rrose Sélavy comme point de rassemblement de différents éléments épars des recherches que je fais depuis plusieurs années sur les vies antérieures. Je me suis promenée dans mes vies antérieures et un peu dans celles de Duchamp. Cela m'a permis de faire une sorte de mise au point sur ce que cette recherche représente. La performance se termine d'ailleurs par une narration de la naissance. Elle est composée de tableaux des différentes mémoires, ce que j’appelle des «time caps», des capsules de temps. Iris Khatchadourian, la narratrice, est un personnage mormon dont j'ai déjà parlé dans mes textes. Elle intervient pour parler de Dieu, du dieu des Mormons, qui ressemble beaucoup au dieu chrétien, mais qui habite près de la planète Kobol. C'est un dieu plus science-fictif, peut-être. Iris Khatchadourian qui vit à Salt Lake City dans les années 1920, va aller avec son père à New York chez les Arensberg où elle rencontre Marcel Duchamp. C'est Duchamp qui parle des vies antérieures dans le texte. Il lui apprend à jouer aux échecs et essaie de lui réveiller la mémoire, lui disant qu'ils se sont connus à l'époque atlante et se demandant comment il se fait qu'elle ne se rappelle pas. Iris Khatchadourian est une jeune fille, une jeune vierge très pudique qui résiste à cette idée en disant: Non, je vais retourner près de Dieu sur la planète Kobol. C'est toute la théorie mormone de la rédemption qui est présentée. Iris Khatchadourian devient comme le modèle à partir duquel Duchamp va créer Rrose Sélavy. Puis une narratrice du présent raconte qu'au cours d'un atelier, elle a découvert ce que représente Marcel Duchamp. C'est l'illumination, mais sous forme d'ironie. Une phrase revient dans la performance: This is the space of miracles. Ceci est l'espace du miracle. Cette performance est un travail sur l'inspiration, sur la force et l'énergie qui nous amènent à la création. Rrose Sélavy représente pour moi l'énergie d'Éros, et l'énergie érotique est aussi l'énergie qui nous pousse à créer.

V. \& I. - Cette recherche que tu fais sur les vies antérieures te sert à quoi?

Y. Villemaire - Je ne sais pas encore. J'ai commencé à m'y intéresser en 1978 au moment où j'étudiais au Mandushri Institute of Tibetan Studies. Nous vivions en commune dans un château au nord de l'Angleterre et je me rappelle qu'une fois, en sortant du monastère, j'ai vu une Prefect, une petite auto anglaise qui avait beaucoup frappé mon imagination quand j'étais enfant. Il fallait la pousser dans les côtes pour qu'elle monte. Je n'en avais jamais revu depuis. Alors j'ai écrit à mon père que j'avais vu la réincarnation 
de sa Prefect. C'était tout ce que je comprenais à la réincarnation. Les bouddhistes avec lesquels je travaillais ne pouvaient pas m'éclairer. Leur conception de la réincarnation se limitait à prêcher la compassion parce que tout le monde peut avoir été notre mère dans une vie antérieure.

En 1981, je suis allée faire un atelier de danse à Maui avec Gabrielle Roth, une chamane américaine avec qui je travaille depuis 1980. C'est là que j'ai rencontré Denise Roussel, qui est parapsychologue à Montréal, et qui est spécialiste des vies antérieures. Je trouvais ça très comique, mais comme j'étais très curieuse, $\mathrm{j}$ 'ai fait un atelier avec elle à mon retour à Montréal à la fin d'août. La technique qu'utilise Denise Roussel est une technique très simple qui lui a été enseignée par le docteur Morris Netherton avec lequel j'ai aussi travaillé. C'est une technique qui lui permet tout simplement de retrouver la mémoire. C'est comme ça que j'ai compris que la réincarnation était une expérience et pas une théorie, une idée. L'intérêt de se rappeler ses vies passées, c'est de pouvoir distinguer ce qui ne relève pas du présent mais qui nous envahit tout de même. Il y a aussi les habiletés qui sont dans le passé et qu'on peut aller chercher. Il y a les blessures qu'on peut guérir, mais il y a aussi le savoir que l'on peut retrouver. Après les ateliers, je me suis rendue compte que ce phénomène m'intéressait depuis toujours. Mon premier souvenir - je n'ai pas un an, je jette ma suce dans le poêle à bois, ça me rappelle quelque chose - est relié à ça. J'ai travaillé spécifiquement sur ce souvenir-là et j'ai trouvé: ça me rappelle de l'autre côté.

Pour une romancière, les vies antérieures constituent un domaine extraordinaire. Il y a plein de personnages, plein d'histoires. C'est une méthode extraordinaire pour aller chercher l'information technique. J'utilise aussi la documentation historique, mais la reconstitution est plus facile par la lecture directe des événements. Il y a juste à aller voir. Je suis convaincue que d'ici à l'an 2000, la notion du fait qu'on a vécu dans le passé et qu'on se réincarne, qu'on meurt et qu'on revient, sera évidente pour tout le monde. Elle fait déjà partie de la réalité quotidienne de la moitié de l'humanité. Nous nous imaginons toujours, nous les occidentaux, être des empereurs du savoir: notre version est qu'on apparaît et qu'on disparaît. Point. C'est vrai aussi, remarque: Yolande Villemaire existe maintenant, elle existe seulement depuis 1949 et elle va mourir je ne sais pas quand, le plus tard possible. Cette identité qui est la mienne n'a jamais été avant moi et ne sera jamais de nouveau. Mais il reste que j'ai été d'autres personnages, qui avaient d'autres personnalités, dont j'ai la mémoire et dont j'ai l'expérience surtout.

Quand on parle de ce sujet, il y a toujours comme une sorte de peur qui est rampante. C'est un sujet miné, difficile, parce que nous avons tous un voile qui nous obscurcit la mémoire. On ne se rappelle pas ses vies antérieures. Je viens de lire Dancing in the Light de Shirley MacLaine. Je ne comprends pas pourquoi la télévision américaine la ridiculise autant. Pourtant, j'avais moi-même cette attitude très ironique avant d'avoir l'expérience des vies antérieures. Maintenant, je me dis que la seule façon de contourner la question est de ne pas en parler. Dans les ateliers d'écriture, d'ailleurs, je n'en parle 
pas. J'amène les gens dans l'expérience immédiatement. Comme ça, il n’y a pas de discussion.

V. \& I. - Ça t'oblige à vivre avec une quantité de personnages, avec toute une série d'egos passés, en plus de ta personnalité actuelle?

Y. Villemaire - Oui, mais ce n'est pas un problème: on le fait de toute façon. J'ai mis trois ans à écrire la Constellation du Cygne. Il a fallu que je fasse beaucoup de détours avant de rassembler toute l'information. En 1981, un atome de mémoire était remonté à la surface: Celia Rosenberg apparaît dans Ange amazone. À l'été 1982, j’ai fait un atelier qui s'appelle «Six Days", donné par Werner Erhard Associates où on nous pousse à notre limite physique. On dort très peu, on mange très peu, on est submergé d'informations, puis on passe une journée à faire de l'escalade et à traverser des précipices. L'imminence du danger est constamment avec nous. C'est au cours de la séance d'entraînement qui précède cette journée en montagne que la mémoire de Celia Rosenberg a émergé. C'était comme une espèce de nuage noir que je n'arrivais pas à cerner. Je voyais des images de l'univers allemand. Je n'aurais pu le raconter, mais je baignais dans l'émotion. J'étais vraiment dans tous mes états quand je suis revenue. J'ai d'abord passé quelques jours chez Gabrielle Roth qui s'est occupée de me déprogrammer. À mon retour à Montréal, j'ai passé une journée en régression avec Denise Roussel. C'est là que la mémoire de Celia Rosenberg s'est organisée dans un ordre linéaire. Une mémoire antérieure, c'est d'abord des impressions, des émotions, des flashes. Denise me faisait parler, je débitais l'histoire. Le soir même, j'entreprenais un nouvel atelier qui s'appelle "Communication for Action" et qui s'adresse surtout aux gens qui travaillent en informatique. Je suis arrivée là complètement survoltée. Je passais d'un état à un autre. À un moment donné, l'ancienne décodeuse en moi a été illuminée. J'ai comme touché le point d'inspiration où Claude Gauvreau se situait. L'atelier se déroulait, les gens parlaient et moi j'écrivais. Parce qu'on s'ennuie toujours un peu dans ces ateliers. J'écrivais en langage exploréen et je comprenais que ce langage avait un sens transmissible. J'ai regardé ma feuille et je me suis dit que non, je n'entrerais pas dans cette dimension-là du langage. Je l'ai quand même fait un peu dans les Coïncidences terrestres, mais l'expérience se limitait à huit mots.

J'avais déjà le nom des personnages. Denise me les avait demandés pendant la régression. J'avais Celia Rosenberg, Piotr Jalski, Karl-Heinz Hausen. J'ai fait le plan de la Constellation. Je voyais les chapitres s'organiser. Mais je ne voulais pas écrire un roman dont l'action se passerait pendant la guerre. Je voulais faire Yvel Swanson, mon grand roman d'espionnage contemporain! J'ai quand même commencé à écrire le 15 août. J'étais à Ogunquit, je me suis assise sur le bord du pier, et j'ai écrit «Paris, 15 août 1940 ». Ça m'a pris deux ans et demi.

C'était la première fois que j'écrivais quelque chose où j'avais le début et la fin. Avec Denise, j'avais vu que le roman se terminait à Auschwitz, mais je n'avais pas d'images précises. Ma vie, de 1982 à 1985 - j'ai terminé le roman 
le 31 décembre 1984 - a donc été de retrouver la mémoire. Le plus difficile a été de retrouver la mémoire de l'éclat de bombe dans la jambe droite. On aurait dit que la seule façon était de repasser par la douleur. J'étais allée à la campagne à la Minerve. Près d'un feu de camp, chez des amis, j'ai rencontré un vieux Belge qui avait été membre des SS noirs et qui me disait qu'il avait été d'accord avec l'holocauste parce que les Juifs étaient une race inférieure. On était en août 1983 au Québec. Il disait ça sans vergogne. J'ai commencé à ávoir mal à la jambe. J'ai passé une semaine à l'hôpital et j'ai traîné pendant un mois et demi. Maintenant je peux voir que cette histoire a libéré l'engramme. Je ne peux plus être l'objet de choses semblables. Avec la Constellation, j'ai atteint une sorte de clarté: je peux identifier ce qui vient du passé.

V. \& I. - Pour un roman dont la gestation a été aussi bouleversante, la Constellation est étonnamment linéaire. Est-ce que de retrouver cette mémoire t'a permis d'exorciser le passé?

Y. Villemaire - Oui. C'est pour cette raison que j'ai pu le couler dans une forme linéaire. La Vie en prose, par exemple, était une expérimentation que je faisais dans tous les sens. Alors que j'ai vraiment écrit la Constellation du Cygne dans un but cathartique, pour me libérer de cette mémoire qui, en fait, était une position de victime. J'ai vécu le roman. J'ai retrouvé des parcelles, des poches d'émotion. En les traversant, j'ai atteint une position mystique, religieuse, qui est reliée à la mort: j’ai atteint le pardon. En écrivant la Constellation du Cygne, je me suis trouvée à pardonner à Hitler. C'est une position inconfortable. Je ne nie pas la souffrance, mais je pense que les erreurs du passé sont là pour nous permettre de ne pas les reproduire. J'ai écrit ce roman aussi parce que je voyais un aveuglement de presque tout le monde à l'époque par rapport à l'holocauste nucléaire. Celia Rosenberg est d'un aveuglement absolument remarquable. Etre Juive, vivre à Paris, en 1940, et ne même pas soupçonner que les Juifs sont tellement menacés! Elle est d'une telle inconscience, d'un tel aveuglement qu'elle contribue malgré elle à ce qui se passe. Aujourd 'hui, on prépare une guerre nucléaire et je suis aussi inconsciente de cela que Celia Rosenberg a pu l'être. Je crois pourtant qu'on peut renverser la situation. Helen Caldicott, qui prononce une conférence dans le film Si cette planète vous tient à cour, dit: Je ne vous parlerais pas de la guerre nucléaire si je pensais qu'on ne peut pas l'empêcher. Je suis comme tout le monde, cependant. Je ne sais pas par quel bout commencer. Les armes nucléaires sont là comme les réactions d'hostilité que l'on éprouve dans nos rapports quotidiens avec les gens. En vivant à New York, je deviens très sensible au fait que l'on peut éprouver une hostilité meurtrière envers quelqu'un qui passe devant soi dans une file au bureau de poste. La vie à New York est plus lourde qu'à Montréal. En même temps, New York est une ville où il y a une grande unité.

V. \& I. - Est-ce que la Constellation marque une transformation chez tes personnages? A-t-elle emporté Yvel Swanson? 
Y. Villemaire - Non. Ce n'est pas du tout une disparition des autres personnages. C'est un retour au passé pour aller vider un abcès. J'aurais pu raconter d'autres abcès. Il y avait un personnage karateka, qui était un homme. Il est passé dans les Coïncidences terrestres, mais il n'a pas fait l'objet de tout un roman. J'ai choisi cet abcès-là parce que peut-être il était le plus évident. Je reporte toujours Yvel Swanson parce que je la saisis mal. La Constellation a peut-être liquidé tout ce qui avait un rapport avec le passé. Yvel Swanson est un personnage qui va vers le futur, vers un monde nouveau, un monde plus unifié. Je ne sais pas de qui, de Vava ou d'Yvel Swanson, le roman que je suis en train de conceptualiser, parlera. Je change d'idée à peu près tous les jours. Vava, c'est la Vava de la Vie en prose. Elle est très contemporaine. Yvel Swanson a un lien avec l'Atlantide et avec le futur. Je vais prendre une décision dans les prochains jours. Je commence à écrire dès mon retour à New York. Cependant, je ne veux pas faire les choses trop vite non plus. Je ne veux pas travailler sur un personnage dont je n'ai pas les racines. Je fais prochainement un atelier qui s'appelle «Power of Ackowledgement ". Si Yvel Swanson émerge saine et sauve de l'atelier, je l'écrirai à ce moment-là. Sinon, je ferai Vava comme transition.

V. \& I. - Tu dis de New York qu'elle est une ville d'une grande unité. C'est pourtant la ville la plus cosmopolite. Comment perçois-tu cette unité?

Y. Villemaire - New York est en effet une ville très cosmopolite. C'est d'abord une ville espagnole. Il y a des vendeurs d'encens sur la rue qui viennent du Kenya et qui parlent leur langue. J'ai rencontré des gens qui viennent d'URSS, de Hollande, de différents endroits. New York est une plaque tournante, un centre international. L'unité vient de là. Elle vient en premier lieu d'une conscience de cette diversité et ensuite du fait que, malgré cette diversité, nous faisons tous partie du même ensemble, notamment de la même ville. Je me rappelle d'une conversation avec une Portoricaine qui avait deux petits garçons, dont l'un était son petit-fils alors que la femme avait à peu près mon âge. Un des garçons venait d'entrer dans le wagon tout seul. Quelqu'un l'avait retenu à temps, autrement il aurait été perdu dans le métro de New York. Nous vivions des vies complètement différentes. En même temps, je comprenais l'état de nervosité dans lequel elle était. Nous avons conversé, parlé des enfants. Nous nous sommes senties en unité. Peutêtre est-ce la conscience de faire partie d'un univers qui est varié, cosmopolite, complexe, où les gens vivent différentes choses, de différents points de vue, où se développe une grande tolérance. Mais en même temps, New York est un mythe pour tout le monde, pour moi qui suis Québécoise, pour le chauffeur de taxi russe qui est là depuis seize ans et qui a un accent épouvantable, pour les Portoricains qui vivent dans le South Bronx. Les gens ont un amour très grand pour cette ville ou alors une haine féroce. Peut-être aussi est-ce parce qu'il y a les Nations Unies qu'il y a cette notion d 'unité. C'est important pour moi que les Nations Unies soient à New York. J'y vais souvent. C'est le seul lieu sur la terre - à part Genève peut-être - qui est un territoire international. Des gens qui y travaillent me disent que j'ai une vision bien poétique des Nations Unies, que dans la pratique, c'est une lourde machine bureaucra- 
tique. C'est vrai. L'intention des Nations Unies est quand même une intention d'unité, de paix, de rassemblement.

V. \& I. - La mémoire dont tu parlais renvoie à des images d'éclatement. Vois-tu dans le futur une espérance d'unité ?

Y. Villemaire - En venant à Montréal, j'ai rencontré dans l'avion un ingénieur en électronique qui m’a montré les nouveaux circuits produits par sa compagnie. Il y en avait une dizaine en or pur sur son carton et il me disait que ces microchips étaient utilisés pour les radars en avionique. C'était fabuleux. Ils peuvent maintenant mettre, je ne sais pas, $1000 \mathrm{~K}$ d'information sur une tête d'épingle. Je lui demandais si le futur pourrait être d'imprimer directement avec notre cerveau, sans utiliser de fil d'or. Il m'a regardée en souriant et a dit peut-être. Je pense que la race humaine est dans une période de mutation, depuis le 4 février 1962, depuis l'entrée dans l'ère du Verseau. Nous avons développé la technologie, la technique, et pourtant nous sommes encore très primitifs au niveau des relations entre deux êtres humains, entre l'être humain et la collectivité, l'être humain et l'ensemble de la planète. Il y a encore des racismes. des sexismes, des choses qui sont vieilles comme le monde et qui sont très arriérées. Cela ne va pas se régler du jour au lendemain, mais je crois que oui, nous allons vers une plus grande unité, vers la conscience que nous sommes tous sur le même bateau, que chacune de nos individualités est une qualité essentielle dans l'ordre du monde.

V. \& I. - Est-ce cette unité que traduit l'image de l'hologramme?

Y. Villemaire - Oui. J'ai l'impression que mon mental est séduit par la notion du même. J'ai été séduite par la notion de gémellité, par exemple. dans mes premiers textes. Mais le même n'est pas nécessairement une identité. Prends l'exemple de la constellation du cygne: chaque étoile y brille de sa propre brillance, de sa propre couleur, mais le cygne visible est créé par l'ensemble des étoiles. Je pense que pour aller vers une plus grande unité, vers une plus grande harmonie, il est très important d'accepter les différences individuelles, que les femmes tolèrent les différences des hommes et que les hommes tolèrent les différences des femmes; que les Blancs tolèrent les différences des Noirs et que les Noirs tolèrent les différences des Blancs. Il y a quelques années, je cherchais plus à niveler pour voir l'unité, comme si j'avais voulu la créer a priori alors qu'elle naît de l'acceptation des différences. C'est comme de vouloir que le bleu soit "le" bleu. Il faut qu'il y ait du turquoise, du bleu pâle, du bleu poudre, du bleu azur, du bleu cobalt...

V. \& I. - A partir de tes recherches, notamment sur les vies antérieures, es-tu arrivée à te percevoir toi-même comme un hologramme, comme un réseau de convergences?

Y. Villemaire - Je t'avoue que je ne sais pas ce qu'est exactement un hologramme. Nicole Brossard l'a amené dans le Texte de la littérature québécoise dès French Kiss. Je me rappelle à l'époque avoir cherché dans le dictionnaire ce qu'était un hologramme. J'avais vu que "holos» veut dire "tout» et 
"gramme», «écriture»: «écriture du tout». J'en ai vu depuis, dans des musées. Je les ai regardés, j’ai passé ma main à travers. Dans «Estelle toute la nuit en elle", je parlais du projecteur hologrammatique, responsable de ma présence ici. C'était une façon d'attribuer la responsabilité à quelqu'un d'autre, mais c'était une façon de me voir comme un point de convergence. Nous sommes la résultante du passé en même temps que la source du futur. C'est cette idée que traduit l'hologramme. La notion d'unité que j'ai, me vient de ma mère. L'unité, c'est sa famille. Cela lui vient de sa mère, ma grand-mère Larose, qui avait dix enfants. Je suis la résultante du projet de ma grand-mère. Je suis la résultante du projet de ma mère. En même temps, je crois sincèrement que nous avons tous les pouvoirs, qu'il s'agit de se libérer de ce qui nous limite, de ce qui nous empêche d'exercer notre liberté totale de créateur. Quel que soit notre travail, nous sommes les créateurs du monde. Malheureusement, les gens qui sont dans le monde matériel ont souvent une sorte d'incompréhension des artistes et des écrivains. Mon père travaille dans la construction. Je ne sais pas s'il a déjà lu mes livres. Il ne m'en a jamais parlé. Pour lui, les mots ne sont rien; ce qui-compte, c'est les fondations de la maison. Les gens qui sont dans les mots, au contraire, trouvent que la matière n'est rien, mais s'ils ne l'avaient pas, ils auraient froid. C'est une autre tolérance à développer. Je trouve que les artistes ne sont pas suffisamment reconnus dans notre société. Il y a comme une espèce de glamour autour de nous, mais notre travail n'est pas reconnu comme tel, comme un travail de conceptualisation, de recherche et d'expérimentation. On ne part pas avec sa boîte à lunch pour aller écrire un roman, mais c'est quand même du travail.

\section{V. \& I. - Tu la vois où, cette importance?}

Y. Villemaire - Les livres ont toujours influencé la réalité, parce que c'est d'abord ce qu'on peut concevoir, conceptualiser, qui va ensuite se réaliser. Si je n'avais jamais pu visualiser un roman écrit, imprimé, avec mon nom dessus, je ne l'aurais jamais écrit. Quand je pense à Yvel Swanson, il faut que je projette l'image d'un livre avec Yvel Swanson sur la couverture, mon nom en dessous et un objet matériel. C'est comme ça que je vais m'asseoir devant ma machine à écrire. En l'écrivant, je vais conceptualiser des choses que je veux pour moi dans le monde et que je veux pour le monde. Je sais que la fiction fait bouger la réalité. Le groupe Rrose Sélavy, c'est les personnages de la Vie en prose, en chair et en os. Ils existent maintenant. Les personnes qui suivent mes ateliers d'écriture sont aussi des personnages devenus réels. Notre importance comme artiste est là. On n'a pas d'importance à soi tout seul, mais avec tous les autres artistes, avec toutes les autres personnes qui agissent sur la réalité.

V. \& I. - Ce travail de conceptualisation peut nous projeter dans deux dimensions différentes: il peut nous projeter vers un programme d'action très concret, ou dans la logique pure, le sacré, l'absolu. Est-ce que tu privilégies l'une ou l'autre?

Y. Villemaire - Quand j'hésite entre Vava et Yvel Swanson, c'est de cela qu'il s'agit. Je m'intéresse de plus en plus à l'aspect sacré, à l'aspect religieux. 
J'ai une relation très importante dans le moment avec une grand-tante, la sœur de ma grand-mère, qui est clarisse à Valleyfield, cloîtrée depuis soixante ans. Elle s'appelle sœur Clémentine. Je suis en relation avec elle depuis la mort de ma grand-mère, l'année dernière. Elle lit mes livres et elle comprend tellement ce que je fais. Il y a une dimension de la contemplation qui est très importante pour moi, mais pas dans cette étape-ci de ma vie. Je suis présentement dans une étape de matérialisation, je veux faire ma part pour changer le monde. J'arrive à l'époque de la maturité. Ma nouvelle idée pour changer le monde est pragmatique: j'aimerais avoir un enfant, une famille. Il y a aussi une action sociale qui va se concrétiser à travers le projet d'un film sur l'Atlantide. Le titre temporaire est To eat - T.E., c'est le contraire de E.T. Je vais le tourner dans le désert arabique en Égypte d'ici cinq ans. Il porte sur une famine à l'époque atlante tout en étant très contemporain. Je voudrais que les revenus aillent aux victimes de la famine en Égypte. J'étais en train d'expliquer que les deux dimensions étaient séparées, mais non, c'est la même chose. La dimension sacrée est là pour ressourcer la dimension plus profane, plus matérialiste, plus pragmatique.

V. \& I. - Tu dis être arrivée à la maturité. Qu'est-ce que tu veux dire par là?

Y. Villemaire - Dans un des ateliers que j'ai fait récemment à New York, il y a eu deux heures d'information sur l'immortalité physique. Il y a des gens qui y croient. J'ai envie de rire comme un bébé chaque fois que j'en entends parler, mais quand j'y pense, je me dis que plus jeune j'étais comme eux. Je n'admettais aucune limite. Je ne faisais pas attention à ma santé, je me sentais invulnérable, je faisais des excès, je fumais beaucoup. Aujourd'hui, je suis plus consciente de mes limites physiques. En même temps, je découvre qu'on peut aller aussi loin que l'on veut. Il y a comme une courbe. Un jour tu arrives à un point qui «est" ta période de production, de productivité dans le monde. J'ai commencé à savoir enseigner il y a cinq ans peut-être et pourtant j'enseigne depuis quatorze ans. C'est la même chose pour l'écriture. J'ai commencé à publier en 1974, mais la Constellation du Cygne est le premier roman dont je sois satisfaite et encore, pas complètement. Il faut des années d'apprentissage. Il faut du temps pour arriver à une sorte de maîtrise. La maturité c'est aussi la responsabilité, la conscience du fait que ce qui se passe est le résultat de nos actions et même de nos pensées.

V. \& I. - Ça suppose alors une sorte de bilan?

Y. Villemaire - J'ai dû le faire. J'ai déménagé à New York le 2 mars et la Constellation paraissait le 4. C'est tellement libérant de finir un projet. J'ai passé mes premières semaines à New York dans les quincailleries de Canal Street pour acheter les choses nécessaires à l'aspect visuel de la performance. J'ai peut-être fait le bilan comme ça, en retrouvant quelque chose qui était en moi quand j'était petite et que j'avais perdu en cours de route. Un jour, j'ai écrit dans mon journal que je croyais dans mon étoile. Mon frère avait dû dire quelque chose qui m'avait fait de la peine. Nous avons tous connu cette période réjouissante de nos vies où on est complètement submergés par le monde, par l'ènergie sexuelle qu'on sent en soi mais qu'on ne comprend pas. 
par les adultes, par la vie. J'ai même écrit des poèmes sur le suicide. Mon étoile était comme ta petite lumière à travers le noir tunnel de la vie. $\mathrm{La}$ constellation du cygne est aussi une étoile. Quand le roman a été publié, j’ai senti que j'avais accompli quelque chose. Je pense aussi à un jeu. Je prenais des cartes géographiques et j'encerclais certaines villes. Je me rappelle avoir encerclé Denver et Dallas. Le réseau télépathique que j'ai créé il y a trois ans, l'Ombre Jaune, est tout à fait la réverbération adulte de ce jeu que je faisais quand j'étais petite, sans comprendre. L'Ombre Jaune aujourd'hui réunit à peu près trois cents membres, au Québec, en Italie, en France et aux ÉtatsUnis, surtout des écrivains, mais aussi des artistes visuels. Je sens que les intuitions que j'avais quand j'étais petite se vérifient. Les fils que j'avais lancés aboutissent après être passés par toutes les montagnes russes de l'adolescence, du début de la jeunesse et de l'âge adulte. Quand on y pense, la vingtaine est une période assez terrifiante. J'ai toujours gardé cela en mémoire pour comprendre les étudiants auxquels je m'adresse. Alors la maturité, c'est de se sentir à sa place, au centre de la vie. J'ai atteint cette sécurité que je n'avais pas avant.

V. \& I. - Tu dis que la Constellation du cygne est la fin de quelque chose. Est-ce qu'on doit considérer Quartz et mica comme un livre transitoire?

Y. Villemaire - La poésie est toujours une transition entre deux romans. Quartz et mica est la fin du cycle. La Constellation est l'avant-fin si l'on veut. "Quartz et mica" d'ailleurs est une sorte de mantra que j'ai entendu pour la première fois en 1978, au moment où je faisais une performance. J'ai utilisé ce son comme générateur. J'avais tout mon temps pour écrire. Je n'avais pas à enseigner et j'avais le studio du Québec à New York qui est un espace merveilleux, un loft blanc, très grand, dans Soho. Je me suis servie de New York comme piscine énergétique. Je me suis traitée comme sujet d'expérience. J'ai laissé la ville m'impressionner, entrer dans mon système physique, mental, émotif, pour opérer une sorte de processing. Mon but était de devenir claire comme du cristal. À l'époque atlante, on utilisait le cristal pour communiquer avec des gens sur d'autres planètes et pour transmettre des pensées. Dans Quartz et mica j'ai voulu nettoyer ce qui restait de la Constellation sans avoir à passer par toutes mes vies. Le livre a été difficile à écrire. J'ai beaucoup résisté à cette énergie. J'ai eu de nombreuses conversations téléphoniques avec Claude Beausoleil qui me disait de laisser le mot et l'image me traverser. Mais moi, je voulais écrire des histoires. Alors j'ai fait plusieurs versions. J'ai raturé, recommencé. J'ai haï ça. La censure était très forte. J'étais en train de me dévaluer complètement comme si je ne faisais plus confiance en mon pouvoir de dire et de comprendre. $\AA$ un moment donné, j'ai pris toutes les critiques que j'ai eues pour la Constellation et je les ai collées au mur. L'ego a fini par lâcher et je me suis décidée à faire mon livre. À l'époque, j'ai eu une conversation téléphonique avec Paul Chamberland. Nous avons parlé d'alchimie. C'est l'or rouge dans Quartz et mica. La Constellation était l'œuvre au noir et Quartz et mica, l'œuvre au rouge. C'est donc la fin d'une étape. Je ne peux pas encore nommer ce qui vient. Je vais pénétrer encore davantage dans la culture. Je vais m'adresser à un public plus vaste. Je sais mieux ce que je fais. Je sais mieux où je vais. 


\section{V. \& I. - Pourquoi veux-tu t'adresser à un public plus vaste?}

Y. Villemaire - Parce que je songe à vivre de mon écriture. L'écriture expérimentale implique qu'on accepte de ne pas vivre de son écriture. Ce travail demeure important et j'y reviendrai peut-être dans des livres de poésie. Pour l'instant, je m'intéresse au travail de recherche des autres. Je pense notamment au travail de Michael Delisle, dont j'ai lu récemment l'Extase neutre. La recherche sur le langage me passionne toujours, mais je sais qu'elle ne passionnerait pas une amie qui est une fanatique de Régine Deforges. Ce qui l'intéresse, c'est comment j'utilise mes recherches. C'est ce que je veux faire maintenant. Claude Beausoleil dit qu'il suffit que je ne laisse pas la parole à Dragan Yedi. Dragan Yedi est un personnage qui revient dans mes textes. C'est une sorte d'Atlante qui mesure sept pieds, qui a les cheveux blonds, les yeux ultra-violets et des manchettes en serpent. Il vient d'une autre planète. Peut-être en effet qu'il ne faut pas que je lui laisse trop d'espace à une époque où je le maîtrise mal. Je n'ai pas intérêt à transmettre des fictions qui sont de l'ordre du laboratoire et que je ne maîtrise pas. On maurait dit cela quand j'écrivais la Vie en prose que j'aurais crié au scandale d'avoir à sacrifier mes personnages. Vouloir atteindre un plus grand public, c'est être responsable de la communication, faire en sorte que le lecteur aille là où je veux l'amener. Cela ne signifie pas qu'on ne lui apporte rien de nouveau. Cela signifie simplement que l'on prend la responsabilité d'organiser tous les éléments d'information de façon à ce qu'un lecteur nous suive. Ce qu'un écrivain comme Marguerite Duras, dans l'Amant, fait remarquablement.

V. \& I. - Tu dis que si on t'avait dit cela, à l'époque de la Vie en prose, tu n'aurais pas accepté de sacrifier des choses pour écrire autrement. Tu as pourtant fait quelque chose comme cela à cette époque. Tu as changé de public aussi.

Y. Villemaire - D'une certaine façon, c'est vrai. Mais c'était autre chose. Avant je censurais presque tout. C'est pour cette raison que mes textes étaient courts. J'étais très prudente, je faisais attention. Je prenais trois semaines à écrire un texte de cinq pages. Claude me rassurait en disant que personne ne lisait Hobo-Québec. J'arrivais à faire mon texte après être passée par des angoisses épouvantables. J'ai mis un an à couper, à ré-écrire Machine-t-elle. Au fond, la seule chose que j'ai sacrifiée, c'est la retenue. La Vie en prose a été comme une débâcle où j'ai laissé tous les personnages venir faire un tour. J'ai été vraiment une très bonne mère pour eux. J'entendais parler de quelque chose et je le mettais dans le roman. C'était une nouvelle attitude. C'est pour cela que j'ai eu beaucoup de plaisir à écrire la Vie en prose.

V. \& I. - Pourtant tu as par la suite abandonné cette débâcle. On n'a plus jamais vu cet éclatement de personnages et de références.

Y. Villemaire - Je n'aime pas réutiliser quelque chose. Je vais toujours ailleurs. Le plus étrange est qu'un de mes projets m'obligerait à réutiliser la Vava de la Vie en prose. En même temps, je vois là une possibilité 
d'intégration. Je vois la possibilité de lui donner une facture plus classique. Les références que tu ramènes sont aussi des questions de langue. Je n'ai jamais réutilisé la langue de la Vie en prose. Au fond, le roman que je voudrais écrire dans le moment serait quelque chose que j'écrirais en français d'Amérique avec facture classique, en réutilisant Vava comme personnage central, en intégrant les recherches d'Ange Amazone sur les vies antérieures et la facture de la Constellation du Cygne. Voilà! C'est un programme très complet. Je veux aussi que ce soit un roman drôle. Il y a un côté de ma personnalité qui est très léger, très joyeux. Il y avait cette énergie dans la Vie en prose, mais parmi un chaos. Le chaos ne m'intéresse plus. Je vais vers quelque chose de plus intégral.

\section{V. \& I. - As-tu une idée de qui sera ton nouveau public?}

Y. Villemaire - J'ai toujours une idée très claire de mon public. Il a toujours été québécois. J'ai écrit la Vie en prose pour les gens qui lisent et qui écrivent. Ce n'était même pas «le» public québécois. La Constellation du Cygne a été écrite pour un public québécois plus large, pas seulement pour les gens qui lisent et qui écrivent. Le roman qui s'appellera Vava ou Yvel Swanson incluerait bien sûr le public québécois, mais aussi le public français auquel je ne me suis jamais adressée. Cela pose d'ailleurs un sérieux problème de langue. Je tiens à écrire comme je parle et je tiens à être comprise en même temps. J'ai mieux réussi à négocier la question du public américain. L'anglais a toujours été une langue importante pour moi. Un peu pour le provoquer, j'ai fait part à Gaston Miron de ma décision d'écrire An American Bestseller. Il a trouvé que l'idée était bonne. J'ai pris ça à la lettre en me disant que je ne serais pas traître à ma nation, même si j'écris en américain. An American Bestseller sera un roman sur les langues. L'Amérique n'est pas seulement les Etats-Unis. Elle comprend le Canada, les Territoires du Nord-ouest, l'Alaska, le Québec, qui est francophone, l'Amérique du Sud, qui est espagnole, et le Brésil, qui est portugais. Le roman sera en anglais à peu près standard, mais avec des tournures syntaxiques assez françaises, des mots en d'autres langues. Il est entendu que la narratrice sera Québécoise et que la réalité québécoise sera partie intégrante du roman. Le public américain implique aussi le public du monde entier. L'anglais m'a toujours fascinée parce que c'est une langue internationale. Mais je ne suis pas encore prête à l'écrire.

\section{V. \& I. - Quel sera le statut de l'espagnol?}

Y. Villemaire - Comme le français, un statut mineur. Un peu comme dans les romans américains. Dans Bright Lights, Big City, de Jim McIwerney, un auteur que j'aime beaucoup, le personnage corrige les épreuves d'un livre en français. Alors il y a des mots français. Dans Ransom, que je viens de lire, c'est un personnage qui est Français. Dans An American Bestseller, le français et l'espagnol seront là pour amener dans l'esprit du lecteur une chose que les Américains ne paraissent pas savoir - ce sont des gens très étranges - qu'il y a d'autres langues que l'anglais. 
V. \& I. - Il y a aussi dans tes livres une écriture régie par d'autres systèmes que les systèmes linguistiques. Qu'est-ce qui va en rester?

Y. Villemaire - Je vais les utiliser comme techniques, un peu comme je vais utiliser les techniques textuelles, la théorie des générateurs et tout ce qui est proprement sémiotique. Je vais les utiliser sans les nommer, sans cet effet de miroir, de métatexte, lequel passionne les gens qui font le travail, mais pas le lecteur qui lui s'intéresse au résultat. Je vais m'en servir au niveau de l'encodage. Ma technique d'écriture, par exemple, est d'utiliser les cartes du tarot. J'utilise un tarot Dakini depuis au moins Ange Amazone. J'ai écrit la Constellation du Cygne avec le même jeu, mais je n'en parle plus. J'envisage d'écrire le prochain roman sans même utiliser ces techniques. En fait, j'utilise des systèmes comme les systèmes cabalistiques pour m'aider à me concentrer. J'ai l'imaginaire très éparpillé. Sans support, je regarde passer les mouches. Je n'écris pas. Les systèmes numérologiques ou le tarot m'aident à concentrer l'énergie, l'inspiration. Je pourrais utiliser n'importe quoi, je pense, comme quand j'étais petite et que j'utilisais les cartes géographiques, les sous, les herbes. J'ai une certaine difficulté avec les fonctions abstraites de la pensée. C'est pour cela que j'aime beaucoup le travail de Nicole Brossard. Elle utilise directement l'abstraction. Claude Beausoleil aussi. Moi je passe par les images. Je vais d'ailleurs me remettre à la peinture et au dessin. Peut-être que si d'une part je fais du dessin et d'autre part j'écris, je pourrai mieux diviser, mieux aller dans l'abstraction. Mais je ne veux pas aller vers l'abstraction comme résultat. Je veux aller vers l'histoire.

V. \& I. - L'histoire?

Y. Villemaire - Oui, comme dans les Mille et une nuits qui sont, je trouve, les plus belles histoires, avec la formule de l'enchâssement, qui est la forme rhétorique qui me plaît le plus. Une histoire dans une histoire dans une histoire... Ça ne finit plus. 